\title{
The Intersection Problem for Finite Semigroups
}

\author{
Lukas Fleischer \\ FMI, University of Stuttgart* \\ Universitätsstraße 38, 70569 Stuttgart, Germany \\ fleischer@fmi.uni-stuttgart.de
}

\begin{abstract}
We investigate the intersection problem for finite semigroups, which asks for a given set of regular languages, represented by recognizing morphisms to finite semigroups, whether there exists a word contained in their intersection. We introduce compressibility measures as a useful tool to classify the intersection problem for certain classes of finite semigroups into circuit complexity classes and Turing machine complexity classes. Using this framework, we obtain a new and simple proof that for groups and commutative semigroups, the problem is contained in NP. We uncover certain structural and non-structural properties determining the complexity of the intersection problem for varieties of semigroups containing only trivial submonoids. More specifically, we prove NP-hardness for classes of semigroups having a property called unbounded order and for the class of all nilpotent semigroups of bounded order. On the contrary, we show that bounded order and commutativity imply containment in the circuit complexity class $\mathrm{qAC}^{k}$ (for some $k \in \mathbb{N}$ ) and decidability in quasi-polynomial time. We also establish connections to the monoid variant of the problem.
\end{abstract}

\section{Introduction}

A careful analysis of the complexity of decision problems for regular languages has triggered renewed interest in the classical intersection non-emptiness problem (called intersection problem in the following), as first described by Kozen in 1977 [11], and in the closely related membership problem for transformation monoids [1, 4, 5, 9, 12]. The connection between these two problems stems from the observation that a set of deterministic finite automata over a common alphabet can be considered as transformations

\footnotetext{
${ }^{*}$ Supported by the German Research Foundation (DFG) under grant DI 435/5-2.
} 
on the (disjoint) union of their states. Both problems are well-known to be PSPACEcomplete in the general case but become easier when the inputs are restricted to have certain structural properties. These properties are often expressed in terms of membership to a certain variety of finite monoids; in the automaton setting, one considers the transition monoids of the automata. For example, for the variety of $\mathcal{R}$-trivial monoids, usually denoted by $\mathbf{R}$, both problems were shown to be decidable in non-deterministic polynomial time [5]. On the other hand, it is known that PSPACE-completeness already holds for any variety not contained within DS, the variety of all finite monoids whose regular $\mathcal{D}$-classes form subsemigroups. However, for many subvarieties of $\mathbf{D S}$, such as $\mathbf{L}$ (the left-right dual of $\mathbf{R}$ ) or DA (all aperiodic monoids from DS), the problems are only known to be NP-hard and to be contained within PSPACE. The problem of determining the exact complexity for varieties in this interval has been open for more than 25 years [5, 15].

Recently, Kufleitner and the author suggested considering the algebraic variant of the problem, where the languages in the input are represented by finite monoids instead of automata [8]. Formally, it is defined as follows:

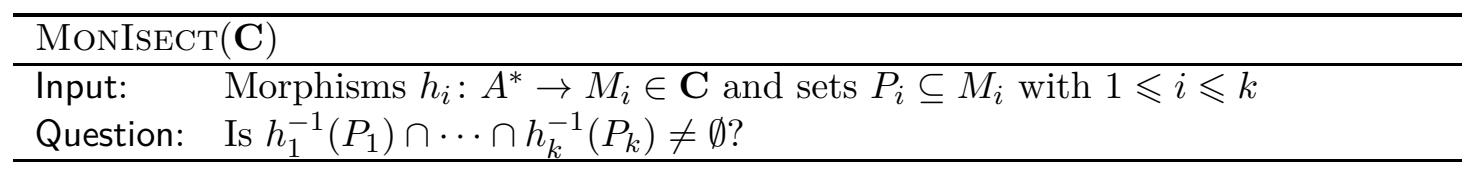

Here, $\mathbf{C}$ is some fixed class of finite monoids and the monoids themselves are given as multiplication tables. Transitioning to the algebraic setting allowed for making some substantial progress in understanding the complexity of the problem: Kufleitner and the author proved NP-completeness of $\operatorname{MonISECT}(\mathbf{D O})$ where DO is a quite large subvariety of DS including both $\mathbf{L}$ and DA. Still, even for the monoid variant, PSPACEcompleteness is only known to hold for varieties not contained within DS, a proper superset of DO.

Attempts to progress further in understanding the complexity of MonIsECT led to the investigation of classes of semigroups $\mathbf{C}$ instead of monoids:

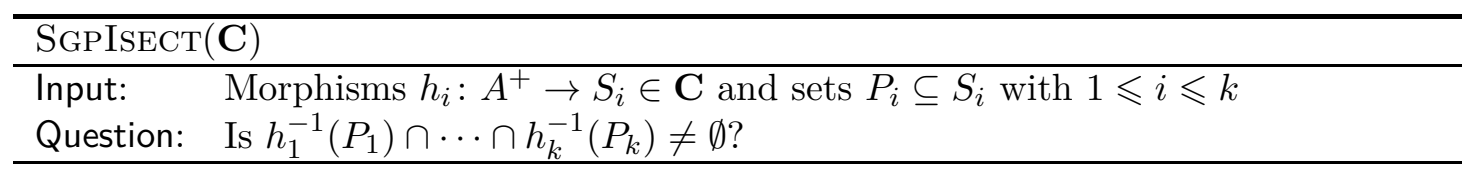

As in the monoid variant, the semigroups are assumed to be given as multiplication tables. While making this distinction between monoids and semigroups may sound subtle at first sight, it has a significant impact on complexity questions and is expected to yield new insights. For example, all known PSPACE-hardness results rely heavily on the existence of neutral letters.

We mainly investigate the intersection problem for varieties of finite semigroups. In [8], $\operatorname{MonIsect}(\mathbf{V})$ was shown to be NP-hard for every non-trivial variety of finite monoids $\mathbf{V}$. Thus, in this work, we focus on the intersection problem for varieties of finite semigroups containing only trivial submonoids. We describe an infinite sequence of varieties $\mathbf{V}_{1} \subseteq$ 
$\mathbf{V}_{2} \subseteq \cdots$ such that $\operatorname{SgPISECT}\left(\mathbf{V}_{i}\right) \in \mathrm{AC}^{0}$ for each $i \geqslant 1$ but the intersection problem for its limit $\operatorname{SgPIsect}\left(\mathbf{V}_{\infty}\right)$ (where $\mathbf{V}_{\infty}=\bigcup_{i \in \mathbb{N}} \mathbf{V}_{i}$ ) is NP-complete. This is surprising for the following reason: for the automaton and monoid variants, all known hardness results are tied to purely structural properties. NP-hardness of MoNIsECT comes from the fact that the problem is NP-hard even for the monoid $U_{1}$ and for the cyclic group $\mathbb{Z} / 2 \mathbb{Z}$, and PSPACE-hardness comes from the fact that even $\operatorname{MonIsECT}\left(B_{2}^{1}\right)$ is PSPACEhard [8]. Since every semigroup from $\mathbf{V}_{\infty}$ is contained in infinitely many varieties $\mathbf{V}_{k}$ in the sequence above, the existence of such a pattern cannot be the sole reason for NPhardness in the semigroup setting. It is open whether a similar situation occurs below DS in the monoid or in the automaton setting.

To investigate other parameters with an impact on the complexity of the problem, we introduce a versatile framework based on the notion of product circuits properties. These properties are a measure of compressibility of witnesses for intersection non-emptiness. Using this framework, we obtain a new and easy proof that both $\operatorname{SgPIseCt}(\mathbf{G})$ and SGPIsect $(\mathbf{C o m})$ are contained in NP. We prove NP-completeness of SGPIsECT for classes having a property we call unbounded order (this includes the class of all nilpotent and commutative semigroups) and for the class of all nilpotent semigroups of bounded order. On the contrary, we show that for every commutative variety with bounded order, the intersection problem is contained in some uniform version of a circuit complexity class $\mathrm{qAC}^{k}$ and thus decidable in quasi-polynomial time. As problems decidable in quasi-polynomial time cannot be NP-hard unless the exponential time hypothesis fails, this suggests that an interplay of structural properties and non-structural properties determines the complexity of the problem. We also suggest a way to transfer complexity results from the monoid setting to the semigroup setting.

\section{Preliminaries}

Algebra. A semigroup is a non-empty set equipped with an associative binary operation, often also referred to as multiplication. A semigroup $M$ with a neutral element, i.e., an element $e \in M$ such that $e x=x=x e$ for all $x \in M$, is called monoid. The neutral element is unique and usually denoted by 1 . An element $x \in S$ is idempotent if $x^{2}=x$ and the set of all idempotent elements of a semigroup $S$ is denoted by $E(S)$. A zero element $z$ of a finite semigroup $S$ satisfies $z x=z=x z$ for all $x \in S$. Each semigroup contains at most one zero element and a semigroup is nilpotent if its only idempotent element is a zero element. The set of all finite words $A^{*}$ (resp. all non-empty finite words $A^{+}$) forms a monoid (resp. semigroup) with concatenation as multiplication.

A subsemigroup (resp. submonoid) of a semigroup (resp. monoid) is a subset closed under multiplication. Let $S$ and $T$ be semigroups and let $M$ and $N$ be monoids. The direct product of $S$ and $T$ is the Cartesian product $S \times T$ with componentwise multiplication. A semigroup morphism from $S$ to $T$ is a mapping $h: S \rightarrow T$ such that $h(s) h(t)=h(s t)$ for all $s, t \in S$. A monoid morphism from $M$ to $N$ is a semigroup morphism $h: M \rightarrow N$ which additionally satisfies $h(1)=1$. The semigroup $T$ is a divisor of $S$ if there exists a surjective semigroup morphism from a subsemigroup of $S$ onto $T$. The monoid $N$ is a 
divisor of $M$ if there exists a surjective monoid morphism from a submonoid of $M$ onto $N$. We often use the term morphism to refer to both semigroup and monoid morphisms if the reference is clear from the context. A morphism $h: A^{+} \rightarrow S$ to a finite semigroup $S$ recognizes a language $L \subseteq A^{+}$if $h^{-1}(P)=L$ for some set $P \subseteq S$. The set $P$ is often called the accepting set for $L$.

Varieties. A variety of finite semigroups is a class of finite semigroups which is closed under taking (semigroup) divisors and direct products. A variety of finite monoids is a class of finite monoids closed under taking (monoid) divisors and direct products. The class $\mathbf{G}$ of all finite groups and the class $\mathbf{I}$ containing only the trivial semigroup $\{1\}$ are both varieties of finite semigroups and varieties of finite monoids. We also consider the following varieties of finite semigroups:

- Com, the variety of all finite commutative semigroups,

- $\mathbf{N}$, the variety of all finite nilpotent semigroups,

- $\mathbf{A}_{2} \cap \mathbf{N}$, the variety of all finite semigroups where $x^{2} y=x^{2}=y x^{2}$ for all $x, y \in S$,

- $\mathbf{L I}_{k}$ (for $k \in \mathbb{N}$ ), the variety of all finite semigroups $S$ which satisfy the equation $x_{1} \cdots x_{k} z y_{k} \cdots y_{1}=x_{1} \cdots x_{k} y_{k} \cdots y_{1}$ for all $x_{1}, \ldots, x_{k}, y_{1}, \ldots, y_{k}, z \in S$.

Note that each of the varieties in this list contains semigroups which are not monoids. Hence, they do not form varieties of finite monoids. We will also briefly refer to the varieties DS and $\mathbf{D O}$ but their formal definitions are not needed.

For a variety of finite semigroups $\mathbf{V}$, we denote by $\mathbf{V}_{\mathbf{M}}$ the class of all finite monoids which, when viewed as semigroups, belong to $\mathbf{V}$. It is easy to check that $\mathbf{V}_{\mathbf{M}}$ forms a variety of finite monoids. For each semigroup $S$ and each idempotent element $e \in E(S)$, the set $e S e$ forms a monoid with the multiplication induced by $S$ and with neutral element $e$, called the local monoid at $e$. For a variety of finite monoids $\mathbf{V}$, we denote by $\mathbf{L V}$ the variety of finite semigroups whose local monoids belong to $\mathbf{V}$. The operators $(\cdot)_{\mathbf{M}}$ and $\mathbf{L}(\cdot)$ are closely related.

Proposition 1 (Folklore). Let $\mathbf{V}$ be a variety of finite monoids and let $\mathbf{W}$ be a variety of finite semigroups. Then $\mathbf{W}_{\mathbf{M}} \subseteq \mathbf{V}$ if and only if $\mathbf{W} \subseteq \mathbf{L V}$. In particular, $\mathbf{W} \subseteq \mathbf{L W}_{\mathbf{M}}$.

Proof. Suppose that $\mathbf{W}_{\mathbf{M}} \subseteq \mathbf{V}$ and let $S$ be an arbitrary semigroup from $\mathbf{W}$. For every idempotent element $e \in E(S)$, the monoid $e S e$ is a subsemigroup of $S$. By closure of $\mathbf{W}$ under taking subsemigroups, we obtain $e S e \in \mathbf{W}$. Since $e S e$ is a monoid, we obtain $e S e \in \mathbf{W}_{\mathbf{M}}$ and by assumption, we have $e S e \in \mathbf{V}$, as desired.

Conversely, suppose that $\mathbf{W} \subseteq \mathbf{L V}$ and let $M$ be a monoid from $\mathbf{W}$. Let $e$ be the identity element of $M$. Since $M \in \mathbf{L V}$, we obtain $M=e M e \in \mathbf{V}$.

As a direct consequence, $\mathbf{L I}$ is the largest variety of finite semigroups not containing any non-trivial monoids. The following proposition connects LI with the hierarchy of varieties $\left(\mathbf{L I}_{k}\right)_{k \in \mathbb{N}}$ defined above. 
Proposition 2 (Folklore). Let $S$ be a finite semigroup of cardinality $n$. Then $S \in \mathbf{L I}$ if and only if $S \in \mathbf{L I}_{n+1}$.

Proof. Suppose that $S \in \mathbf{L I}$ and let $x_{1}, \ldots, x_{n+1}, y_{1}, \ldots, y_{n+1}, z \in S$. By the pigeon hole principle, there exist indices $i, i^{\prime} \in\{1, \ldots, n+1\}$ such that $i<i^{\prime}$ and $x_{1} \cdots x_{i}=x_{1} \cdots x_{i^{\prime}}$. Thus, $x_{1} \cdots x_{i} e=x_{1} \cdots x_{i}$ for $e=\left(x_{i+1} \cdots x_{i^{\prime}}\right)^{\omega}$ and for every $\omega \in \mathbb{N}$. In particular, we may choose $\omega$ such that $e$ is idempotent. Analogously, there exists some index $j \in\{1, \ldots, n+1\}$ and some idempotent element $f$ such that $f y_{j} \cdots y_{1}=y_{j} \cdots y_{1}$. Since $S \in \mathbf{L I}$, we have $e x f=e x(f e f)=(e x f e) f=e f=(e y f e) f=e y(f e f)=e y f$ for all $x, y \in S$. This yields

$$
\begin{aligned}
x_{1} \cdots x_{n+1} z y_{n+1} \cdots y_{1} & =x_{1} \cdots x_{i} e x_{i+1} \cdots x_{n+1} z y_{n+1} \cdots y_{j+1} f y_{j} \cdots y_{1} \\
& =x_{1} \cdots x_{i} e x_{i+1} \cdots x_{n+1} y_{n+1} \cdots y_{j+1} f y_{j} \cdots y_{1} \\
& =x_{1} \cdots x_{n+1} y_{n+1} \cdots y_{1}
\end{aligned}
$$

which shows that $S \in \mathbf{L I}_{n+1}$.

Conversely, let $S$ be contained in $\mathbf{L I}_{n+1}$. For all $e \in E(S)$ and for all $x \in S$, we have exe $=e^{n+1} x e^{n+1}=e^{n+1} e^{n+1}=e$ where only the second equality uses $S \in \mathbf{L I}_{n+1}$. Thus, every local monoid $e S e$ is trivial, and $S \in \mathbf{L I}$.

Complexity. We assume familiarity with standard definitions from circuit complexity. A function has quasi-polynomial growth if it is contained in $2^{\mathcal{O}\left(\log ^{c} n\right)}=n^{\mathcal{O}\left(\log ^{c-1} n\right)}$ for some fixed $c \in \mathbb{N}$. Throughout the paper, we denote by $\mathrm{AC}^{k}\left(\right.$ resp. $\mathrm{qAC}^{k}$ ) the class of languages decidable by circuit families of depth $\mathcal{O}\left(\log ^{k} n\right)$ and polynomial size (resp. quasi-polynomial size); see [3, 14, 16] for details. We allow NOT gates but do not count them when measuring the depth or the size of a circuit. We will also refer to the standard complexity classes NP and PSPACE. The exponential time hypothesis states that a deterministic Turing machine cannot decide 3-SAT in subexponential time. If the hypothesis is true, NP-complete problems cannot be decided in quasi-polynomial time; see e.g. [10].

Straight-Line Programs. A straight-line program $(S L P)$ is a tuple $G=\left(V, A, P, X_{s}\right)$ where $V$ is a finite set of variables, $A$ is a finite set of letters, $P: V \rightarrow(V \cup A)^{*}$ is a mapping and $X_{s} \in V$ is the so-called start variable such that the relation

$$
\left\{(X, Y) \mid P(X) \in(V \cup A)^{*} Y(V \cup A)^{*}\right\}
$$

is acyclic. For a variable $X \in V$, the word $P(X)$ is the right-hand side of $X$. Starting with some word $\alpha \in(V \cup A)^{*}$ and repeatedly replacing variables $X \in V$ by $P(X)$ yields a word from $A^{*}$, the so called evaluation of $\alpha$, denoted by $\operatorname{val}(\alpha)$. The word produced by $G$ is $\operatorname{val}(G)=\operatorname{val}\left(X_{s}\right)$. If the reference to $A$ and $V$ is clear, we will often use the notation $h(\alpha)$ instead of $h(\operatorname{val}(\alpha))$ for the image of the evaluation of a word $\alpha \in(A \cup V)^{*}$ under a morphism $h: A^{+} \rightarrow S$. Analogously, we write $h(G)$ instead of $h(\operatorname{val}(G))$. The size of $G$ is $|G|=\sum_{X \in V}|P(X)|$. Each variable $X$ of an SLP $G$ can be viewed as an SLP itself by making $X$ the start variable of $G$. 
The canonical SLP of a word $w \in A^{+}$is $G=\left(V, A, P, X_{s}\right)$ with $V=\left\{X_{s}\right\}$ and $P\left(X_{s}\right)=$ $w$. The following simple lemma illustrates how SLPs can be used for compression; see e.g. [6, 8] for a proof.

Lemma 3. Let $G=\left(V, A, P, X_{s}\right)$ be an $S L P$ and let $e \in \mathbb{N}$. Then there exists an SLP $H$ of size $|H| \leqslant|G|+4 \log (e)$ such that $\operatorname{val}(H)=(\operatorname{val}(G))^{e}$.

\section{Product Circuits Properties}

Let $\mathbf{C}$ be a class of finite semigroups and let $f: \mathbb{N} \rightarrow \mathbb{N}$ be a monotonically increasing function. We say that $\mathbf{C}$ has the $f(n)$ circuits property if for each morphism $h_{i}: A^{+} \rightarrow S$ to a finite semigroup $S \in \mathbf{C}$ and for each $w \in A^{+}$, there exists an SLP $G$ of size at most $f(|S|)$ such that $h(G)=h(w)$. We say that $\mathbf{C}$ has the $f(n)$ product circuits property if for each set of morphisms $h_{i}: A^{+} \rightarrow S_{i}$ to finite semigroups $S_{1}, \ldots, S_{k} \in \mathbf{C}$ and for each $w \in A^{+}$, there exists an SLP $G$ of size at most $f\left(\left|S_{1}\right|+\cdots+\left|S_{k}\right|\right)$ such that $h_{i}(G)=h_{i}(w)$ for all $i \in\{1, \ldots, k\}$. For a class of functions $\mathcal{C}$, we say that $\mathbf{C}$ has the $\mathcal{C}$ circuits property (resp. $\mathcal{C}$ product circuits property) if $\mathbf{C}$ has the $f(n)$ circuits property (resp. $f(n)$ product circuits property) for some $f \in \mathcal{C}$.

Let us introduce some abbreviations for commonly used classes of functions. We will use the terms

- constant circuits property and constant product circuits property (const $C P$ and const $P C P$, in short) for the class of constant functions, i.e., the class of all functions of the form $f(n)=c$ for some $c \in \mathbb{N}$,

- poly-logarithmic circuits property and poly-logarithmic product circuits property (polylog $C P$ and polylog $P C P$, in short) for the class of poly-logarithmic functions, i.e., the class of all functions $f(n)=\log ^{c} n$ for some $c \in \mathbb{N}$, and

- polynomial circuits property and polynomial product circuits property (poly CP and poly $P C P$, in short) for the class of polynomials, i.e., the class of all functions of the form $f(n)=n^{c}$ for some $c \in \mathbb{N}$.

The intuition behind these concepts is as follows. The $f(n)$ circuits property is a compressibility measure for witnesses of non-emptiness of a language given by a recognizing morphism. The $f(n)$ product circuits property is a compressibility measure for witnesses of non-emptiness of intersections of languages given by recognizing morphisms. The terminology is inspired by the poly-logarithmic circuits property which was introduced in [7]: having the $f(n)$ circuits property is equivalent to requiring every element of a subsemigroup $S$ of a semigroup from the class to be computable by an algebraic circuit of size $f(n)$ over any set of generators of $S$. Analogously, having the $f(n)$ product circuits property can be expressed in terms algebraic circuits with multiplication gates for the direct product of semigroups. It is clear that the $f(n)$ product circuits property implies the $f(n)$ circuits property. For the other direction, a weaker statement holds. 
Proposition 4. Let $\mathbf{C}$ be a class of finite semigroups which is closed under taking direct products and has the $f(n)$ circuits property. Then $\mathbf{C}$ has the $f\left(n^{n}\right)$ product circuits property.

Proof. Suppose we are given morphisms $h_{i}: A^{+} \rightarrow S_{i}$ to finite semigroups $S_{1}, \ldots, S_{k} \in \mathbf{C}$ and a word $w \in A^{+}$. Let $N=\left|S_{1}\right|+\cdots+\left|S_{k}\right|$. Every semigroup contains at least one element, so $N^{N} \geqslant N^{k}$ is an upper bound for the product $\left|S_{1}\right| \cdots\left|S_{k}\right|$.

Let $S$ be the direct product $S_{1} \times \cdots \times S_{k}$ and let $h: A^{+} \rightarrow S$ be the morphism defined by $h(a)=\left(h_{1}(a), \ldots, h_{k}(a)\right)$ for all $a \in A$. By closure of $\mathbf{C}$ under taking direct products, we have $S \in \mathbf{C}$. Since $\mathbf{C}$ has the $f(n)$ circuits property, there exists some SLP $G$ of size at most $f(|S|)=f\left(\left|S_{1}\right| \cdots\left|S_{k}\right|\right) \leqslant f\left(N^{N}\right)$ such that $h(G)=h(w)$. By construction, $h_{i}(G)=h_{i}(w)$ for all $i \in\{1, \ldots, k\}$.

An essential ingredient in the proof of $\operatorname{MonIseCt}(\mathbf{D O}) \in \mathrm{NP}$ is that the variety of finite groups $\mathbf{G}$ has the poly PCP. In [8], this was verified by analyzing a variant of the Schreier-Sims algorithm. Using the previous proposition, we obtain a much simpler proof: it is well known - and easy to show - that $\mathbf{G}$ has the polylog $\mathrm{CP}$, a result often called the Babai-Szemerédi Reachability Lemma [2]. The statement then follows from the following corollary of Proposition 4.

Corollary 5. Let $\mathbf{C}$ be a class of finite semigroups which is closed under taking direct products and has the polylog $C P$. Then $\mathbf{C}$ has the poly $P C P$.

The corollary also implies that the variety of all commutative semigroups, which was shown to have the polylog $\mathrm{CP}$ in [7, has the poly PCP.

Circuits properties and product circuits properties have a big impact on the complexity of the so-called Cayley semigroup membership problem and the intersection problem for a given class. The remainder of this section is devoted to establishing this link for product circuits properties.

Lemma 6. Let $h: A^{+} \rightarrow S$ be a morphism to a finite semigroup $S$ of size $N$ and let $G$ be an SLP of size $m$ over $A$. Then there exists an unbounded fan-in Boolean circuit of size $m\left(N^{2}+|A|+2\right)\lceil\log N\rceil$ and depth $2 m+2$ which computes $h(G)$. Given the SLP, this circuit can be computed by a deterministic Turing machine in time polynomial in the circuit size.

Proof. Single multiplications can be performed by circuits of size $\left(N^{2}+1\right)\lceil\log N\rceil$ with one layer of AND gates and one layer of OR gates: to perform a multiplication of two elements $x$ and $y$, we need to extract the $\lceil\log N\rceil$-bit entry of the multiplication table in row $x$ and column $y$. We create a layer of $N^{2}\lceil\log N\rceil$ AND gates, followed by a layer of $\lceil\log N\rceil$ OR gates. Each AND gate is connected to one bit of the multiplication table in the input and to all bits of the values $x$ and $y$. Some of the incoming wires corresponding to the values $x$ and $y$ are negated such that the AND gate copies the bit of the multiplication table if it belongs to the corresponding entry $(x, y)$ and evaluates to 0 otherwise. In the second layer, there are $\lceil\log N\rceil$ OR gates. The $k$-th of these OR gates is fed with the outputs of all AND gates corresponding to the $k$-th bit of some 
multiplication table entry. Thus, there are $N^{2}$ incoming wires to each OR gate. Since, for given input values $x$ and $y$, at most one of the incoming wires to each OR gate evaluates to 1 , the result of the product $x \cdot y$ then clearly appears as output value of the OR gates.

A very similar layout is used to lookup the image of a letter $a \in A$ under the morphism $h: A^{+} \rightarrow S$. First, $|A|\lceil\log N\rceil$ AND-gates are used to zero out the images of all letters except for the image of the letter $a$. Then, we use $\lceil\log N\rceil$ OR gates to perform a bitwise OR of all these preprocessed images. Since all images except $h(a)$ are zeroed out, the result is $h(a)$, as desired.

We evaluate the image of each of the variables bottom-up: for all letters $a \in A$ occurring in $G$ we first compute the image $h(a)$. Then, if $P(X)=\gamma_{1} \cdots \gamma_{\ell}$ for some $\gamma_{1}, \ldots, \gamma_{\ell} \in V \cup A$ and the images $h\left(\gamma_{1}\right), \ldots, h\left(\gamma_{\ell}\right)$ have already been computed, we compute $h(X)=h\left(\gamma_{1}\right) \cdots h\left(\gamma_{\ell}\right)$ by performing $\ell-1$ multiplications.

Clearly, each "lookup gadget", each multiplication gadget and the wires connecting these components can be computed by a deterministic Turing machine in time polynomial in the size of the resulting circuit.

We are now able to prove the main result of this section.

Theorem 7. Let $\mathbf{C}$ be a class of finite semigroups with the $f(n)$ product circuits property. Then $\operatorname{SgPISECT}(\mathbf{C})$ is decidable by a family of unbounded fan-in Boolean circuits of size $\mathcal{O}\left((f(n)+n)^{(f(n))^{2}} f(n) n^{3} \log n\right)$ and depth $\mathcal{O}(f(n))$. For each input size $n \in \mathbb{N}$, the corresponding circuit can be computed by a deterministic Turing machine in time polynomial in the size of the resulting circuit.

Proof. Suppose we are given morphisms $h_{i}: A^{+} \rightarrow S_{i}$ to finite semigroups $S_{i} \in \mathbf{C}$ and sets $P_{i} \subseteq S_{i}$ where $1 \leqslant i \leqslant k$ for some $k \in \mathbb{N}$. We let $N=\left|S_{1}\right|+\cdots+\left|S_{k}\right|$. Note that if $n$ denotes the input size of the SGPIsEct instance, we have $N \leqslant n$ and $|A| \leqslant n$. Since C has the $f(n)$ product circuits property, we know that if there exists a word $w \in A^{+}$ such that $h_{i}(w) \in P_{i}$ for all $i \in\{1, \ldots, k\}$, then this word is generated by some SLP of size at most $f(N) \leqslant f(n)$.

First, note that for a given fixed SLP of size $f(n)$, we can compute the image of the word generated by the SLP under each of the morphisms by an unbounded fan-in Boolean circuit of size $\mathcal{O}\left(n f(n) n^{2} \log n\right)$ and depth $\mathcal{O}(f(n))$ by Lemma 6. Since there are at most $\left((f(n)+n)^{f(n)}\right)^{f(n)}$ different SLPs of size $f(n)$ - at most $f(n)$ variables and at most $(f(n)+n)^{f(n)}$ possible right-hand sides per variable - we can do this evaluation for each of the SLPs in parallel, check whether any of them produces a witness for intersection non-emptiness and feed the outcomes of all the circuits into a single OR gate. It is clear that an enumeration of all SLPs of size at most $f(n)$ can be realized by a deterministic Turing machine in time polynomial in the output size.

For classes with the const PCP and classes with the polylog PCP, efficient decidability of SGPISECT is an immediate consequence.

Corollary 8. Let $\mathbf{C}$ be a class of finite semigroups with the const PCP. Then the decision problem $\operatorname{SGPISECT}(\mathbf{C})$ is contained in $\mathrm{AC}^{0}$. 
Corollary 9. Let $\mathbf{C}$ be a class of finite semigroups with the polylog PCP. Then the decision problem $\operatorname{SgPISECT}(\mathbf{C})$ is contained in $\mathrm{qAC}^{k}$ for some $k \in \mathbb{N}$. Moreover, it is decidable in quasi-polynomial time and thus not NP-hard, unless the exponential time hypothesis fails.

Proof. Containment in $\mathrm{qAC}^{k}$ is an immediate consequence of Theorem[7, For decidability in quasi-polynomial time, we can use a Turing machine that first computes and then evaluates the circuit. The circuit evaluation is done by computing the output value of a gate whenever all its inputs are available.

For the poly PCP, the statement of Theorem 7 only yields exponential-size circuits. We restate a more useful complexity result on poly PCP classes from 8 .

Theorem 10. Let $\mathbf{C}$ be a class of finite semigroups with the poly $P C P$. Then $\operatorname{SgPISECT}(\mathbf{C})$ is contained in NP.

Proof. We proceed as in the proof of Theorem 7 but instead of generating a circuit evaluating all SLPs of polynomial size in parallel, we non-deterministically guess only one such SLP. We then evaluate the corresponding circuit in polynomial time as described in Lemma 6.

Together with the observations above, we obtain an easy proof of containment of both $\operatorname{SgPIseCt}(\mathbf{G})$ and $\operatorname{SgPIseCT}(\mathbf{C o m})$ in NP.

Even though product circuits properties are a powerful tool, in some cases, it is sufficient to consider short witnesses without compression. This is particularly true for varieties not containing any subgroups which we shall mostly be concerned with in the following section. Moreover, for the const PCP, compressibility and the existence of short (non-compressed) witnesses are actually equivalent.

Proposition 11. A class of finite semigroups $\mathbf{C}$ has the const PCP if and only if there exists some constant $\ell \in \mathbb{N}$ such that every non-empty intersection of languages recognized by semigroups from $\mathbf{C}$ contains a word of length at most $\ell$.

Proof. The direction from right to left is trivial: every word $w$ of length at most $\ell$ can be represented by its canonical SLP, which then has size at most $\ell$ as well.

For the converse direction, suppose that there exists some $s \in \mathbb{N}$ such that every non-empty intersection contains a word generated by an SLP of size at most $s$. It is easy to see that the length of such a word is at most $s^{s}$ : there are at most $s$ variables and the right-hand side of every variable has length at most $s$; the claim now follows by induction. Thus, we obtain the desired statement by setting $\ell=s^{s}$.

\section{The Intersection Problem for Locally Finite Semigroups}

Before presenting any algorithms and hardness results for SGPISECT, let us first describe how to transfer existing results to the semigroup setting. 
Proposition 12. Let $\mathbf{V}$ be a variety of finite semigroups. If $\mathbf{V} \nsubseteq \mathbf{L I}$, then $\operatorname{SgPIseCt}(\mathbf{V})$ is NP-hard. If $\mathbf{V} \nsubseteq \mathbf{L D S}$, then $\operatorname{SgPISECT}(\mathbf{V})$ is PSPACE-hard.

Proof. If $\mathbf{V} \nsubseteq \mathbf{L I}$, then $\mathbf{V}_{\mathbf{M}} \nsubseteq \mathbf{I}$. Therefore, by [8, Theorem 8], MonIsect $\left(\mathbf{V}_{\mathbf{M}}\right)$ is NP-hard. The claim now follows from the fact that $\operatorname{Mon} \operatorname{IsECT}\left(\mathbf{V}_{\mathbf{M}}\right)$ is trivially $\mathrm{AC}^{0}$-reducible to $\operatorname{SgPIseCt}(\mathbf{V})$. The same technique allows lifting PSPACE-hardness of $\operatorname{MonIsect}\left(\mathbf{V}_{\mathbf{M}}\right)$ in the case $\mathbf{V}_{\mathbf{M}} \not \subset \mathbf{D S}[\underline{8}$, Theorem 11].

It seems plausible that the $\mathbf{L}(\cdot)$ operator can be used to lift complexity results from MonIsect to SGPIseCt in a more general way. We thus conjecture:

Conjecture 13. If MonIsect $(\mathbf{V})$ is in NP, then $\operatorname{SgPIsect}(\mathbf{L V})$ is in NP.

By [8], a proof of this conjecture would immediately yield that SGPIseCT(LDO) is contained in NP. A possible approach is making use of the fact that for a local variety of finite monoids $\mathbf{V}$, we have $\mathbf{L V}=\mathbf{V} * \mathbf{D}$; see e.g. [13] for details. However, one also needs to account for the size of semigroups from $\mathbf{V} * \mathbf{D}$. Surprisingly, even lifting the group case is much harder than one would expect. Our attempts to adapt the group algorithm from [8] failed and it is known from [7] that LG does not have the polylog CP, so we cannot use Corollary 5 as in the group case.

To summarize, up to this point, the complexity landscape of SGPISECT looks as follows. By Proposition 12, the problem is NP-hard for every variety $\mathbf{V} \nsubseteq \mathbf{L I}$. Using the DOalgorithm from [8], we know that the problem is NP-complete for every variety $\mathbf{V} \subseteq \mathbf{D O}$ not contained within LI. For $\mathbf{V} \nsubseteq \mathbf{L D S}$, the problem is PSPACE-complete. This leaves two classes of varieties for further investigation:

1. For $\mathbf{V} \nsubseteq \mathbf{D O}$ and $\mathbf{V} \subseteq \mathbf{L D S}$, we do not know whether the problem is always NP-complete, whether it becomes PSPACE-complete for varieties contained within LDS already and whether any other classes inside LDS are connected to natural complexity classes, such as the polynomial hierarchy.

2. Thus far, we do not have any hardness results for $\mathbf{V} \subseteq \mathbf{L I}$.

The remainder of this section is devoted to the second class of varieties. On one hand, it is not difficult to see that $\operatorname{SGPIseCT}(\mathbf{L I})$ is contained in NP. On the other hand, NP-hardness holds only for some subvarieties of LI but not for others. Containment in NP actually already follows from $\mathbf{L I} \subseteq \mathbf{D O}$ but it also is an immediate consequence of the following result.

Theorem 14. For each $k \geqslant 1$, the variety $\mathbf{L I}_{k}$ has the $2 k$ product circuits property. In particular, $\operatorname{SgPISECT}\left(\mathbf{L} \mathbf{I}_{k}\right)$ is contained in $\mathrm{AC}^{0}$.

Proof. It suffices to show that for each $k \in \mathbb{N}$ and for each finite semigroup $S \in \mathbf{L I}_{k}$, each morphism $h: A^{+} \rightarrow S$ and each $u=a_{1} \cdots a_{\ell} \in A^{+}$with $\ell>2 k$, the word $v=$ $a_{1} \cdots a_{k} a_{\ell-k+1} \cdots a_{\ell}$ satisfies $h(v)=h(u)$. To see this, note that

$$
\begin{aligned}
h(v) & =h\left(a_{1} \cdots a_{k} a_{\ell-k+1} \cdots a_{\ell}\right)=h\left(a_{1}\right) \cdots h\left(a_{k}\right) h\left(a_{\ell-k+1}\right) \cdots h\left(a_{\ell}\right) \\
& =h\left(a_{1}\right) \cdots h\left(a_{k}\right) h\left(a_{k+1} \cdots a_{\ell-k}\right) h\left(a_{\ell-k+1}\right) \cdots h\left(a_{\ell}\right)=h\left(a_{1} \cdots a_{\ell}\right)=h(u)
\end{aligned}
$$


where the third equality holds by the definition of $\mathbf{L I}_{k}$. The length of $v$ is $|v|=k+(\ell-$ $(\ell-k))=2 k$. Since the word $v$ does not depend on $h$ or on $S$, the canonical SLP of $v$ yields the desired product circuits property.

Combining Theorem 14 with Proposition 2, we immediately obtain that LI has the $2 n+2$ product circuits property: each of the semigroups $S_{1}, \ldots, S_{k}$ in the input has cardinality at most $N=\left|S_{1}\right|+\cdots+\left|S_{k}\right|$. Hence, all semigroups $S_{i}$ belong to the variety $\mathbf{L I}_{N+1}$ and there exists a witness of size at most $2 N+2$.

Corollary 15. The variety $\mathbf{L I}$ has the poly $P C P$. In particular, $\operatorname{SgPISECT}(\mathbf{L I})$ is contained in NP.

Another consequence of Proposition 2 is $\bigcup_{k \in \mathbb{N}} \mathbf{L I}_{k}=\mathbf{L I}$. For each variety in the infinite sequence $\mathbf{L I}_{1} \subseteq \mathbf{L I}_{2} \subseteq \cdots$, the intersection problem is in $\mathrm{AC}^{0}$ but for its limit $\mathbf{L I}$, the problem is only contained in NP - and it is actually NP-complete, as we shall see later. Therefore, in contrast to previously obtained hardness results which relied on purely structural properties, other parameters interfere with the complexity of SGPISECT below LI. We will investigate this phenomenon more carefully. A semigroup is monogenic if it is generated by a single element. The order of a class $\mathbf{C}$ of finite semigroups is the supremum of the cardinalities of all monogenic subsemigroups contained in $\mathbf{C}$. If the order is $\infty$, the class is said to have unbounded order. The following observation will be used implicitly several times later.

Lemma 16 (Folklore). Let $S$ be a finite semigroup from $\mathbf{L I}$ and let $s \in S$. Then there exists some integer $n \in \mathbb{N}$ such that for all $i \in \mathbb{N}$, we have $s^{n+i}=s^{n}$. This integer is the order of the monogenic subsemigroup generated by s. Moreover, if $S$ is nilpotent, then $s^{n}$ is the zero element.

Proof. Since $S$ is finite, there exist $n \in \mathbb{N}$ and $p \geqslant 1$ with $s^{n}=s^{n+p}$. Let $n$ and $p$ be minimal with this property. If $p>1$, then $s^{n p+1}$ generates a non-trivial subgroup of $S$, a contradiction to the assumption that $S \in \mathbf{L I}$. Thus $p=1$, yielding the first part of the statement.

It is clear that $s^{2 n}=s^{n+n}=s^{n}$, thus $s^{n}$ is idempotent. Since in a nilpotent semigroup, the only idempotent element is a zero element, we obtain the desired statement.

In follow-up results, we will use reductions from 3-SAT to prove NP-hardness of SGPIsECT for varieties of semigroups with certain properties. To simplify notation, let us introduce some definitions. For a set of variables $X=\left\{x_{1}, \ldots, x_{k}\right\}$, we let $\bar{X}=\{\bar{x} \mid x \in X\}$ where each $\bar{x}$ is a new symbol. The set of literals over $X$ is $X \cup \bar{X}$ and a set of literals is a clause. An assignment $\mathcal{A}: X \rightarrow\{0,1\}$ of truth values to the variables $X$ can be extended to all literals over $X$ by letting $\mathcal{A}(\bar{x})=1-\mathcal{A}(x)$ and to clauses $C \subseteq X \cup \bar{X}$ by letting $\mathcal{A}(C)=\max \{\mathcal{A}(\ell) \mid \ell \in C\}$. An assignment $\mathcal{A}$ satisfies a set of clauses $\left\{C_{1}, \ldots, C_{n}\right\}$ if $\mathcal{A}\left(C_{j}\right)=1$ for all $j \in\{1, \ldots, n\}$. For a word $w \in(X \cup \bar{X})^{+}$, the mapping $\mathcal{A}_{w}: X \rightarrow\{0,1\}$ defined by $\mathcal{A}_{w}(\ell)=1$ if and only if $w \in(X \cup \bar{X})^{*} \ell(X \cup \bar{X})^{*}$ for all $\ell \in X \cup \bar{X}$ is called the assignment induced by $w$. Note that this assignment is well-defined whenever $\{w\} \cap(X \cup \bar{X})^{*} x_{i}(X \cup \bar{X})^{*} \cap(X \cup \bar{X})^{*} \overline{x_{i}}(X \cup \bar{X})^{*}=\emptyset$ for all 
$i \in\{1, \ldots, k\}$. Conversely, for a given assignment $\mathcal{A}: X \rightarrow\{0,1\}$, we call $w_{\mathcal{A}}=\ell_{1} \cdots \ell_{k}$, where $\ell_{i}=x_{i}$ if $\mathcal{A}\left(x_{i}\right)=1$ and $\ell_{i}=\overline{x_{i}}$ otherwise, the word induced by $\mathcal{A}$.

Theorem 17. If $\mathbf{V}$ is a variety of finite semigroups with unbounded order, then the decision problem $\operatorname{SgPISECT}(\mathbf{V})$ is NP-hard.

Proof. We may assume $\mathbf{V} \subseteq \mathbf{L I}$, otherwise $\operatorname{SgPIsect}(\mathbf{V})$ is NP-hard by Proposition 12 , For each $k \in \mathbb{N}$ the semigroup $S_{k}=\{1, \ldots, k\}$ with the binary operation $i \circ j=$ $\min \{i+j, k\}$ is contained in $\mathbf{V}$. To see this, take some arbitrary $k \in \mathbb{N}$. Since $\mathbf{V}$ has unbounded order, some monogenic semigroup $T$ of cardinality $m \geqslant k$ appears as a subsemigroup in $\mathbf{V}$. Let $s$ be a generator of $T$. By Lemma [16] and since $m \geqslant k$, the mapping $h: T \rightarrow S_{k}$ defined by $h(s)=1$ is a surjective morphism. By closure of $\mathbf{V}$ under divisors, the semigroup $S_{k}$ itself belongs to $\mathbf{V}$.

We now reduce 3-Sat to $\operatorname{SgPISEct}(\mathbf{V})$. Suppose we are given a set of variables $X=\left\{x_{1}, \ldots, x_{k}\right\}$ and a set of clauses $\left\{C_{1}, \ldots, C_{n}\right\}$ where $C_{j}=\left\{\ell_{j 1}, \ell_{j 2}, \ell_{j 3}\right\}$ for each $j \in\{1, \ldots, n\}$ and for literals $\ell_{j 1}, \ell_{j 2}, \ell_{j 3}$ over $X$.

We let $S=S_{k+2}$ be the monogenic semigroup of cardinality $k+2$ defined above. We introduce morphisms $g_{0}, \ldots, g_{k}, h_{1}, \ldots, h_{n}:(X \cup \bar{X})^{+} \rightarrow S$ defined by

$$
g_{i}(\ell)=\left\{\begin{array}{ll}
2 & \text { if } i>0 \text { and } \ell \in\left\{x_{i}, \overline{x_{i}}\right\}, \\
1 & \text { otherwise }
\end{array} \quad h_{j}(\ell)= \begin{cases}2 & \text { if } \ell \in C_{j}, \\
1 & \text { otherwise }\end{cases}\right.
$$

for $0 \leqslant i \leqslant k$ and $1 \leqslant j \leqslant n$. We let $P_{0}=\{k\}, P_{1}=\cdots=P_{k}=\{k+1\}$ and $Q_{1}=\cdots=Q_{n}=\{k+1, k+2\}$. It is easy to check that the intersection

$$
L=\bigcap_{i=0}^{n} g_{i}^{-1}\left(P_{i}\right) \cap \bigcap_{j=1}^{k} h_{j}^{-1}\left(Q_{j}\right)
$$

is non-empty if and only if there exists a satisfying assignment. To see this, the following three observations are crucial:

1. $g_{0}^{-1}\left(P_{0}\right)$ contains all words over $(X \cup \bar{X})$ with exactly $k$ letters,

2. $g_{i}^{-1}\left(P_{i}\right) \cap g_{0}^{-1}\left(P_{0}\right)$ contains all words from the set $(X \cup \bar{X})^{k}$ with exactly one occurrence of $x_{i}$ or exactly one occurrence of $\overline{x_{i}}$ (but not both), and

3. $h_{j}^{-1}\left(Q_{j}\right) \cap g_{0}^{-1}\left(P_{0}\right)$ contains all words from the set $(X \cup \bar{X})^{k}$ with at least one occurrence of any of the literals $\ell_{j 1}, \ell_{j 2}, \ell_{j 3}$.

By the first two properties, all words from $L$ are of the form $\ell_{1} \cdots \ell_{k} \in(X \cup \bar{X})^{k}$ with $\left|\left\{\ell_{1}, \ldots, \ell_{k}\right\} \cap\left\{x_{i}, \overline{x_{i}}\right\}\right|=1$ for all $i \in\{1, \ldots, k\}$. Thus, for each $w \in L$, the assignment $\mathcal{A}_{w}$ induced by $w$ is well-defined.

Now, if $w \in L$, by the third property, we have $\mathcal{A}_{w}\left(\ell_{j 1}\right)=1$ or $\mathcal{A}_{w}\left(\ell_{j 2}\right)=1$ or $\mathcal{A}_{w}\left(\ell_{j 3}\right)=1$ for each $j \in\{1, \ldots, n\}$. Thus, $\mathcal{A}_{w}$ is satisfying. Conversely, if there exists a satisfying assignment $\mathcal{A}: X \rightarrow\{0,1\}$, the word induced by $\mathcal{A}$ is contained in $L$.

It is obvious that the reduction can be performed in polynomial time. A more careful analysis shows that the reduction can even be carried out by a $A C^{0}$ circuit family. 
To complement the previous result, let us now consider a very restricted variety of order 2 (one can show that all varieties $\mathbf{V} \subseteq \mathbf{L I}$ of order 1 are so-called rectangular bands and contained in $\mathbf{L} \mathbf{I}_{1}$ already).

Theorem 18. SgpIsect $\left(\mathbf{A}_{2} \cap \mathbf{N}\right)$ is NP-complete.

Proof. As in the previous proof, we reduce 3-SAt to $\operatorname{SgpIsect}\left(\mathbf{A}_{2} \cap \mathbf{N}\right)$. Containment in NP follows from Corollary 15 and from $\mathbf{A}_{2} \cap \mathbf{N} \subseteq \mathbf{N} \subseteq \mathbf{L I}$.

Suppose we are given a set of variables $X=\left\{x_{1}, \ldots, x_{k}\right\}$ as well as a set of clauses $\left\{C_{1}, \ldots, C_{n}\right\}$ where $C_{j}=\left\{\ell_{j 1}, \ell_{j 2}, \ell_{j 3}\right\}$ for each $j \in\{1, \ldots, n\}$ and literals $\ell_{j 1}, \ell_{j 2}, \ell_{j 3}$ over $X$. Let $S$ be the finite semigroup $\{(i, j) \mid 1 \leqslant i \leqslant j \leqslant k\} \cup\{0\}$ defined by the multiplication

$$
(i, j)(k, \ell)= \begin{cases}(i, \ell) & \text { if } k=j+1 \\ 0 & \text { otherwise }\end{cases}
$$

The element 0 is a zero element. Let $g, h_{1}, \ldots, h_{n}:(X \cup \bar{X})^{+} \rightarrow S$ be the morphisms defined by $g\left(x_{i}\right)=g\left(\overline{x_{i}}\right)=(i, i)$ and by

$$
h_{j}\left(x_{i}\right)=\left\{\begin{array}{ll}
(i, i) & \text { if } x_{i} \notin C_{j}, \\
0 & \text { otherwise },
\end{array} \quad h_{j}\left(\overline{x_{i}}\right)= \begin{cases}(i, i) & \text { if } \overline{x_{i}} \notin C_{j}, \\
0 & \text { otherwise. }\end{cases}\right.
$$

for $1 \leqslant i \leqslant k$ and $1 \leqslant j \leqslant n$. As accepting sets, we choose $P=\{(1, k)\}$ for $g$ and $Q_{1}=\cdots=Q_{n}=\{0\}$ for $h_{1}, \ldots, h_{n}$. Again, we would like to show that the intersection

$$
L=g^{-1}(P) \cap \bigcap_{j=1}^{k} h_{j}^{-1}\left(Q_{j}\right)
$$

is non-empty if and only if there exists a satisfying assignment for $\left\{C_{1}, \ldots, C_{n}\right\}$. The following two properties hold:

1. $g^{-1}(P)$ contains all words of the form $\ell_{1} \cdots \ell_{k}$ with $\ell_{i} \in\left\{x_{i}, \overline{x_{i}}\right\}$ for $1 \leqslant i \leqslant k$,

2. $g^{-1}(P) \cap h_{j}^{-1}\left(Q_{j}\right)$ contains all words of this form containing at least one of the letters $\ell_{j 1}, \ell_{j 2}, \ell_{j 3}$.

Let $w \in A^{+}$be a word with $g(w) \in P$ and $h_{j}(w) \in Q_{j}$ for all $j \in\{1, \ldots, n\}$. Then, by the first property above, the assignment $\mathcal{A}_{w}$ induced by $w$ is well-defined. Moreover, by the second property, we have $\mathcal{A}_{w}\left(C_{1}\right)=\cdots=\mathcal{A}_{w}\left(C_{n}\right)=1$ and thus, $\mathcal{A}_{w}$ satisfies $\left\{C_{1}, \ldots, C_{n}\right\}$. Conversely, it is easy to see that each word induced by a satisfying assignment is contained in $L$.

Note that the constructed semigroup belongs to $\mathbf{A}_{2} \cap \mathbf{N}$ since by definition, we have $(i, j)(i, j)=0$ for all $(i, j) \in S$.

It is obvious that the reduction can be performed in polynomial time.

In view of the previous theorems, the following result might be surprising. For the class of all commutative semigroups within LI, the semigroup intersection problem is NP-hard by Theorem 17. The variety $\mathbf{A}_{2} \cap \mathbf{N}$ has order 2 and its semigroup intersection problem is NP-hard by Theorem 18. However, if we combine commutativity and bounded order, the problem becomes easier. 
Theorem 19. If $\mathbf{V} \subseteq \mathbf{C o m} \cap \mathbf{L I}$ is a variety of finite semigroups with bounded order, then $\mathbf{V}$ has the polylog PCP. Thus, there exists some $k \in \mathbb{N}$ such that $\operatorname{SGPISECT}(\mathbf{V}) \in \mathrm{qAC}^{k}$ and $\operatorname{SGPISECT}(\mathbf{V})$ is decidable in quasi-polynomial time.

Proof. We show that if every monogenic subsemigroup of $S \in \mathbf{C o m} \cap \mathbf{L I}$ has size at most $k$, then every product of at least $k(\log |S|+1)$ elements is the zero element. Thus, every non-empty intersection of languages recognized by multiple morphisms to such semigroups contains a witness of logarithmic size. Note that $\mathbf{C o m} \cap \mathbf{L I} \subseteq \mathbf{N}$, so the $k$-fold power of any element in $S$ is the zero element.

Assume, for the sake of contradiction, that there exists a product of at least $k(\log |S|+$ 1) elements which is not the zero element. By reordering elements, we can rewrite this product as $s_{1}^{i_{1}} \cdots s_{m}^{i_{m}}$ with $s_{i} \neq s_{j}$ for $1 \leqslant i<j \leqslant m$. We proceed by induction on $m$. If $m \leqslant \log |S|+1$, then there exists some $r \in\{1, \ldots, m\}$ with $i_{r} \geqslant k$. Since each monogenic subsemigroup of $S$ has size at most $k$, the element $s_{r}^{i_{r}}$ then is a zero element, a contradiction. Suppose now that $m>\log |S|+1$.

The set $T=\mathcal{P}(\{1, \ldots, m\}) \backslash\{\emptyset\}$ forms a semigroup with union as binary operation. Let $h: T \rightarrow S$ be the morphism defined by $h(r)=s_{r}^{i_{r}}$ for $1 \leqslant r \leqslant m$. We have $|T|=2^{m}-1 \geqslant 2^{m-1}>2^{\log |S|}=|S|$. Thus, by the pigeon hole principle, there exist two sets $K_{1}, K_{2} \subseteq\{1, \ldots, m\}$ with $K_{1} \neq K_{2}$ and $h\left(K_{1}\right)=h\left(K_{2}\right)$.

If $K_{1} \subsetneq K_{2}$, then multiplying the product by $h\left(K_{2} \backslash K_{1}\right)$ does not change its value and $k$-fold multiplication shows that the product is zero, a contradiction. The case $K_{2} \subsetneq K_{1}$ is symmetric. Thus, we may assume that neither $K_{1} \subseteq K_{2}$ nor $K_{2} \subseteq K_{1}$. The length of a set $K \subseteq\{1, \ldots, m\}$ is the sum of all $i_{r}$ with $r \in K$. By symmetry, we may assume that the length of $K_{1}$ is at most the length of $K_{2}$. We replace the factor $h\left(K_{1}\right)$ of the product by $h\left(K_{2}\right)$ and obtain the statement by induction on the number $m$ of different elements in the product - the length of this new product $h\left(K_{2}\right) h\left(\{1, \ldots, m\} \backslash K_{1}\right)$ is at least the length of the original product and the number of different elements decreases since $K_{1} \backslash K_{2} \neq \emptyset$.

\section{Open Problems}

It remains open whether the observation that hardness is not always caused by purely structural properties also applies to varieties between LI and LDS in the semigroup setting, between DO and DS in the monoid setting or between $\mathbf{R}$ and DS in the automaton setting. Another major challenge is obtaining algebraic characterizations of all classes of finite semigroups with the poly PCP. As a first step, we suggest proving (or disproving) that the variety $\mathbf{L G}$ has the poly PCP.

Acknowledgements. I would like to thank the anonymous referees of the conference version of this paper for providing helpful comments. 


\section{References}

[1] L. Babai, E. M. Luks, and Á. Seress. Permutation groups in NC. In STOC 1987, Proceedings, pages 409-420, 1987.

[2] L. Babai and E. Szemeredi. On the complexity of matrix group problems I. In 25th Annual Symposium on Foundations of Computer Science, pages 229-240, Oct 1984.

[3] D. A. M. Barrington. Quasipolynomial size circuit classes. In Proceedings of the Seventh Annual Structure in Complexity Theory Conference, pages 86-93, Jun 1992.

[4] M. Beaudry. Membership Testing in Transformation Monoids. PhD thesis, McGill University, Montreal, Quebec, 1988.

[5] M. Beaudry, P. McKenzie, and D. Thérien. The membership problem in aperiodic transformation monoids. J. ACM, 39(3):599-616, 1992.

[6] M. Charikar, E. Lehman, D. Liu, R. Panigrahy, M. Prabhakaran, A. Sahai, and A. Shelat. The smallest grammar problem. IEEE Transactions on Information Theory, 51(7):2554-2576, July 2005.

[7] L. Fleischer. On the Complexity of the Cayley Semigroup Membership Problem. In CCC 2018, Proceedings, pages 25:1-25:12. Dagstuhl Publishing, 2018.

[8] L. Fleischer and M. Kufleitner. The intersection problem for finite monoids. In STACS 2018, Proceedings, pages 30:1-30:14. Dagstuhl Publishing, 2018.

[9] M. Furst, J. Hopcroft, and E. Luks. Polynomial-time algorithms for permutation groups. In SFCS 1980, Proceedings, pages 36-41, Oct 1980.

[10] R. Impagliazzo and R. Paturi. Complexity of k-SAT. In CCC 1999, Proceedings, pages 237-240, 1999.

[11] D. Kozen. Lower bounds for natural proof systems. In FOCS 1977, Proceedings, pages 254-266, Providence, Rhode Island, 1977. IEEE Computer Society Press.

[12] C. C. Sims. Computational methods in the study of permutation groups. In Proceedings of the Conference on Computational Problems in Abstract Algebra 1967, Oxford, United Kingdom, pages 169-183, New York, 1968. Pergamon.

[13] H. Straubing. Finite semigroup varieties of the form $\mathbf{V} * \mathbf{D}$. Journal of Pure and Applied Algebra, 36(1):53-94, 1985.

[14] H. Straubing. Finite Automata, Formal Logic, and Circuit Complexity. Birkhäuser, Boston, Basel and Berlin, 1994.

[15] P. Tesson and D. Thérien. Diamonds are forever: The variety DA. In Semigroups, Algorithms, Automata and Languages 2001, Proceedings, pages 475-500. World Scientific, 2002. 
[16] H. Vollmer. Introduction to Circuit Complexity. Springer, Berlin, 1999. 